\title{
Hyperfinite-Dimensional Representations of Canonical Commutation Relation
}

\author{
Hideyasu Yamashita \\ Graduate School of Human Informatics, \\ Nagoya University, Nagoya 464-01, Japan
}

\begin{abstract}
This paper presents some methods of representing canonical commutation relations in terms of hyperfinite-dimensional matrices, which are constructed by nonstandard analysis. The first method uses representations of a nonstandard extension of finite Heisenberg group, called hyperfinite Heisenberg group. The second is based on hyperfinite-dimensional representations of $s o(3)$. Then, the cases of infinite degree of freedom are argued in terms of the algebra of hyperfinite parafermi oscillators, which is mathematically equivalent to a hyperfinite-dimensional representation of $s o(n)$.
\end{abstract}

PACS numbers: 03.65.-w 


\section{INTRODUCTION}

In the field of mathematical foundations of quantum physics, especially of infinite degree of freedom, it is often argued that we need any extensions of separable Hilbert space as the fundamental mathematical tools, such as the linear space of generalized functions.

In recent years, another alternative, nonstandard-analytical extension method, is developed by some authors ${ }^{1,2,3,4}$. The present paper adopts an approach in use of hyperfinite-dimensional linear spaces, constructed by nonstandard analysis $^{5,6}$ (see Appendix to find the basic definitions).

This approach also is closely related with that of finite-dimensional quantum physics ${ }^{7,8,9,10}$; nonstandard extensions of finite-dimensional models in quantum physics turn out to be the powerful tools for the approach.

The purpose of the present paper is representing canonical commutation relasion $(\mathrm{CCR})[Q, P]=i$ (let us take the natural unit $\hbar=1$ ), or equivalently, $\left[a, a^{\dagger}\right]=1$ where $a=(Q+i P) / \sqrt{2}$, in a hyperfinite-dimensional linear space. Let $\nu$ be an infinite hypernatural number, and $Q$ and $P$ internal $\nu \times \nu$ hermitian matrices. $Q$ and $P$ act on the $\nu$-dimensional internal linear space ${ }^{\star} \mathbf{C}^{\nu}$ as internal linear operators. No standard finite-dimensional matrices satisfy CCR. By the transfer principle in nonstandard analysis, the fact also holds for any internal hyperfinite-dimensional matrices, so we have $[Q, P] \neq i$. Thus we must arrange the standard CCR for hyperfinite-dimensional matrices. First we consider the following weaker version of $\mathrm{CCR}:[Q, P] \approx i$, that is, $\|[Q, P]-i\|_{\infty} \approx 0$, where $\|A\|_{\infty}$ is given by

$$
\|A\|_{\infty}=\sup \left\{\|A \xi\|:\|\xi\| \leq 1, \xi \in{ }^{\star} \mathbf{C}^{\nu}\right\}
$$

This is equivalent to the condition that for any $\xi \in{ }^{\star} \mathbf{C}^{\nu}$ with finite norm, $[Q, P] \xi \approx i \xi$ ( i.e., $\|([Q, P]-i) \xi\| \approx 0$ where $\|\cdot\|$ is the norm on ${ }^{\star} \mathbf{C}^{\nu}$ defined by $\left.\left\|\left(\xi_{1}, \ldots, \xi_{\nu}\right)\right\|=\sqrt{\xi_{1}^{2}+\cdots+\xi_{\nu}^{2}}\right)$. We see that the condition never holds as follows. Suppose the condition holds. Then any eigenvalue $\lambda$ of $[Q, P]$ satisfies $\lambda \approx i$. This contradicts $\operatorname{Tr}([Q, P])=0$. Now, let us define the still weaker notion of representation of CCR by limiting the domain of the operators $Q$ and $P$.

Definition 1.1: Let $Q_{k}, P_{k}$ be $\nu \times \nu$ internal hermitian matrices ( $k=$ $1,2, \ldots<\infty)$, and $S$ be an external subspace of ${ }^{\star} \mathbf{C}^{\nu}$ which consists only of the vectors with finite norm. The set of the triples $\left(Q_{k}, P_{k}, S\right)$ (or of pairs

$\left(a_{k}, S\right)$ where $\left.a_{k}=\left(Q_{k}+i P_{k}\right) / \sqrt{2}\right)$ is called a quasi-representation of CCR if

$$
\left[Q_{k}, P_{l}\right] \xi \approx i \delta_{k l} \xi,\left[Q_{k}, Q_{l}\right] \xi \approx\left[P_{k}, P_{l}\right] \xi \approx 0
$$

for any $\xi \in S$ and $k, l=1,2, \ldots<\infty$. A quasi-representation of CCR $\left(Q_{k}, P_{k}, S\right)$ is called a representation of $C C R$ if $S$ is invariant respect to $Q_{k}, P_{k}$ (i.e., $Q_{k} S, P_{k} S \subseteq S$ ). 
Hereafter, we will use the following notations.

$(1)^{\star}$ is the mapping of nonstandard extension.

(2) $\mathbf{N}\left({ }^{\star} \mathbf{N}\right)$ is the set of natural (hypernatural) numbers.

(3) $\mathbf{Z}\left({ }^{\star} \mathbf{Z}\right)$ is the set of integers (hyperintegers).

(4) $\mathbf{R}\left({ }^{\star} \mathbf{R}\right)$ is the set of real (hyperreal) numbers.

(5) $\mathbf{C}\left({ }^{\star} \mathbf{C}\right)$ is the set of complex (hypercomplex) numbers.

\section{CANONICAL PAIR OF UNITARY MA- TRICES}

Definition 2.1: Let $M\left({ }^{\star} \mathbf{C}, \nu\right)$ denote the set of internal $\nu \times \nu$ matrices $\left(\nu \in{ }^{\star} \mathbf{N}\right)$. Unitary matrices $U, V \in M\left({ }^{\star} \mathbf{C}, \nu\right)$ are called a canonical pair if $U^{k}, V^{k} \neq 1(k=1, \ldots, \nu-1), U^{\nu}=V^{\nu}=1$ and $U V=e^{2 \pi i / \nu} V U$.

Let $\left|u_{0}\right\rangle$ be a normalized eigenvector of $U$ with eigenvalue $c_{0}$.

Lemma 2.1: For any $n \in{ }^{\star} \mathbf{N}, V^{n}\left|u_{0}\right\rangle$ is an eigenvector of $U$, and its eigenvalue is $e^{2 \pi n i / \nu} c_{0}$.

Proof: Induction for $n$. $U V^{n+1}\left|u_{0}\right\rangle=U V V^{n}\left|u_{0}\right\rangle=e^{2 \pi i / \nu} V U V^{n}\left|u_{0}\right\rangle=$ $e^{2 \pi i / \nu} V e^{2 \pi n i / \nu} c_{0} V^{n}\left|u_{0}\right\rangle=c_{0} e^{2 \pi(n+1) i / \nu} V^{n+1}\left|u_{0}\right\rangle$. Q.E.D.

From the definition, we find that all the eigenvalues of $U$ are $e^{2 \pi i / \nu}, e^{4 \pi i / \nu}, \ldots, e^{2 \nu \pi i / \nu}=1$. Let us assume $c_{0}=1$.

Lemma 2.2: Define $\left|u_{k}\right\rangle$ and $\left|v_{n}\right\rangle$ by $\left|u_{k}\right\rangle=V^{k}\left|u_{0}\right\rangle$ and $\left|v_{n}\right\rangle=$ $\frac{1}{\sqrt{\nu}} \sum_{k=0}^{\nu-1} e^{2 \pi n k i / \nu}\left|u_{k}\right\rangle\left(k, n \in{ }^{\star} \mathbf{N}\right) .\left|v_{n}\right\rangle$ is an eigenvector of $V$, and its eigenvalue is $e^{-2 \pi n i / \nu}$.

Proof: $V\left|v_{n}\right\rangle=\frac{1}{\sqrt{\nu}} \sum_{k=0}^{\nu-1} e^{2 \pi n k i / \nu} V^{k+1}\left|u_{0}\right\rangle=$ $e^{-2 \pi n i / \nu} \frac{1}{\sqrt{\nu}} \sum_{k=0}^{\nu-1} e^{2 \pi n k i / \nu} V^{k}\left|u_{0}\right\rangle=e^{-2 \pi n i / \nu}\left|v_{n}\right\rangle$. Q.E.D.

From these lemmas, we see that $\left|u_{k}\right\rangle$ and $\left|v_{k}\right\rangle(k=0, \ldots, \nu-1)$ are complete orthonormal systems of ${ }^{\star} \mathbf{C}^{\nu}$. Thus we have

$$
\begin{gathered}
V=\sum_{k=0}^{\nu-1}\left|u_{k+1}\right\rangle\left\langle u_{k}\left|=\sum_{k=0}^{\nu-1} e^{-2 \pi n i / \nu}\right| v_{n}\right\rangle\left\langle v_{n}\right| \\
U=\sum_{k=0}^{\nu-1}\left|v_{k+1}\right\rangle\left\langle v_{k}\left|=\sum_{k=0}^{\nu-1} e^{2 \pi n i / \nu}\right| u_{n}\right\rangle\left\langle u_{n}\right|
\end{gathered}
$$

The following proposition follows from these consequences.

Proposition 2.3: The canonical pair of $\nu \times \nu$ unitary matrices is unique in the sense of unitary equivalence, that is, if $(U, V)$ and $\left(U^{\prime}, V^{\prime}\right)$ are two such canonical pairs, then there is a unitary matrix $W$ such that $U^{\prime}=W U W^{\dagger}, V^{\prime}=W V W^{\dagger}$.

Definition 2.2: Let $A \in M\left({ }^{\star} \mathbf{C}, \nu\right) . \quad|a\rangle \in{ }^{\star} \mathbf{C}^{\nu}$ is called approximately invariant vector of $A$ iff $\langle a \mid a\rangle<\infty$ and $A|a\rangle \approx|a\rangle$. 
Let $I(A)$ denote the set of all the approximately invariant vectors of $A$. $I(A)$ is an external subspace of ${ }^{\star} \mathbf{C}^{\nu}$.

Theorem 2.4: If $\nu>\infty$, then $I(U) \cap I(V)$ is an invariant subspace of $U$ and $V$, and is infinite-dimensional (i.e., it has an infinite orthonormal system), and

$$
\frac{\nu}{2 \pi m n}\left[U^{m}, V^{n}\right]|a\rangle \approx i|a\rangle .
$$

holds for any $|a\rangle \in I(U) \cap I(V)$ and $m, n \in \mathbf{Z}$.

Proof: $I(U) \cap I(V)$ is clearly an invariant subspace. Suppose $l=0,1, \ldots<$ $\infty, \mu>\infty$ and $\mu / \nu \approx 0$. Define $|l\rangle$ by

$$
|l\rangle=\frac{1}{\sqrt{\mu}} \sum_{k=l \mu}^{(l+1) \mu-1}\left|u_{k}\right\rangle
$$

$\{|0\rangle,|1\rangle, \ldots\}$ is an orthonormal system of $I(U) \cap I(V)$. In fact, $\| V|l\rangle-|l\rangle \|^{2}=$ $(1 / \mu) \|\left|u_{(l+1) \mu}\right\rangle-\left|u_{l \mu}\right\rangle \|^{2} \approx 0$, and $\| U|l\rangle-|l\rangle \|^{2}=(1 / \mu) \sum_{k=l \mu}^{(l+1) \mu-1} \mid e^{2 \pi \mu i / \nu}-$ $\left.1\right|^{2} \approx 0$. We have also $(\nu / 2 \pi m n)\left(U^{m} V^{n}-V^{n} U^{m}\right)|a\rangle=\nu / 2 \pi m n\left(e^{2 \pi m n i / \nu}-\right.$ 1) $V^{n} U^{m}|a\rangle \approx i V^{n} U^{m}|a\rangle \approx i|a\rangle$. Q.E.D.

Corollary: Suppose $m, n \in \mathbf{N}$. Let $P^{(m)}, Q^{(n)}$ be hermitian matrices defined by

$$
P^{(m)}=\frac{i}{m} \sqrt{\frac{\nu}{8 \pi}}\left(U^{m}-U^{-m}\right), \quad Q^{(n)}=\frac{i}{n} \sqrt{\frac{\nu}{8 \pi}}\left(V^{n}-V^{-n}\right)
$$

If $|a\rangle \in I(U) \cap I(V)$, then

$$
\left[Q^{(n)}, P^{(m)}\right]|a\rangle \approx i|a\rangle
$$

and hence, $\left(Q^{(n)}, P^{(m)}, I(U) \cap I(V)\right)$ is a quasi-representation of CCR for each $m$ and $n$.

We will give some other properties of a canonical pair of unitary matrices.

Definition 2.3: Let $K \in \mathbf{N}$. Let $\mathbf{K}$ be the ring of residue classes of $\mathbf{Z}$ modulo $K$, i.e., $\mathbf{K}=\mathbf{Z} / K \mathbf{Z}$. For $k, k^{\prime} \in \mathbf{K}, k \oplus k^{\prime}$ stands for the sum of $k$ and $k^{\prime}$ in $\mathbf{K}, k \otimes k^{\prime}$ the product of $k$ and $k^{\prime}$, and $\ominus k$ the minus $k$. The finite Heisenberg group based on $\mathbf{K}$ is the group $H_{K}$ with the underlying set $\mathbf{K} \times \mathbf{K} \times \mathbf{K}$ whose group operation is

$$
(k, l, m)\left(k^{\prime}, l^{\prime}, m^{\prime}\right)=\left(k \oplus k^{\prime}, l \oplus l^{\prime}, m \oplus m^{\prime} \oplus\left(k \otimes l^{\prime}\right)\right) .
$$

The notion of Hyperfinite Heisenberg group is the nonstandard (internal) extension of that of finite Heisenberg group; its definition is given by substituting ${ }^{\star} \mathbf{N}$ and ${ }^{\star} \mathbf{Z}$ for $\mathbf{N}$ and $\mathbf{Z}$ in the above definition, respectively.

Suppose $\nu>\infty$. The canonical pair $U, V$ generates an internal group $G$. We see

$$
G=\left\{e^{2 \pi i m / \nu} V^{l} U^{k} \mid k, l, m=0,1, \ldots, \nu-1\right\}
$$


The following property is easily seen.

Proposition 2.5: Define the mapping $\pi: H_{\nu} \rightarrow M\left({ }^{\star} \mathbf{C}, \nu\right)$ by $\pi(k, l, m)=$ $e^{2 \pi i m / \nu} V^{l} U^{k} \cdot \pi$ is an internal irreducible unitary representation of the hyperfinite Heisenberg group $H_{\nu}$.

Consider $\mathbf{R}^{3}$ with coordinates $(p, q, t)$. We make $\mathbf{R}^{3}$ into a locally compact group with group law

$$
(p, q, t)\left(p^{\prime}, q^{\prime}, t^{\prime}\right)=\left(p+p^{\prime}, q+q^{\prime}, t+t^{\prime}+p q^{\prime}\right) .
$$

We call this group the Heisenberg group and denote it by $H$. The Schödinger representation of $H$ is the storong continuous unitary representation $\rho$ of $H$ on $L^{2}(\mathbf{R})$ defined by

$$
(\rho(p, q, t) f)(x)=e^{2 \pi i(t+q x)} f(x+p)
$$

for all $f \in L^{2}(\mathbf{R})$. This is a realization of Weyl's commutation relation $U(q) V(p)=e^{i q p} V(p) U(q)$ where $U(q)$ and $V(p)$ are one-parameter unitary groups. From the representation of $H_{\nu}$, Ojima and Ozawa ${ }^{1}$ constructed a representation of $H$ in a hyperfinite-dimensional Hilbert space which is unitary equivalent to the Schödinger representation of $H$. By using this representation, they found a nonstandard-analytical proof of noncommutative Parseval's identity

$$
\iint_{\mathbf{R}^{2}}\left\langle\psi_{1}|\rho(p, q, 0)| \phi_{1}\right\rangle\left\langle\phi_{2}\left|\rho(p, q, 0)^{-1}\right| \psi_{2}\right\rangle d p d q=\left\langle\phi_{2} \mid \phi_{1}\right\rangle\left\langle\psi_{1} \mid \psi_{2}\right\rangle,
$$

for all $\psi_{i}, \phi_{i} \in L^{2}(\mathbf{R})(i=1,2)$.

\section{III. $S O(3)$-REPRESENTATION OF CCR}

Standard position and momentum operators $x, p$ with $[x, p]=i$ have the $x-p$ rotation covariant property, that is,

$$
x \cos \theta+p \sin \theta=e^{i \theta H} x e^{-i \theta H}
$$

where $H$ is given by $x^{2}+p^{2}$. This property is essential when dealing with quantum harmonic oscillators. On the other hand, $Q^{(n)}$ and $P^{(m)}$ defined above have no clear covariant property of this sort. This fact reveals a defect of these matrices. In this section, we argue another hyperfinite-dimensional representation of CCR, which have the clear rotation covariance.

Let $J_{1}, J_{2}$ and $J_{3}$ be an irreducible set of $(p+1) \times(p+1)$ hermitian matrices which satisfies

$$
\left[J_{k}, J_{l}\right]=i \epsilon_{k l m} J_{m}
$$


where $\epsilon_{k l m}$ is Levi-Civita symbol. $i J_{1}, i J_{2}$ and $i J_{3}$ generate a $p+1$ dimensional irreducible representation of Lie algebra $s o(3)$. It is known that $J_{k}$ has eigenvalues

$$
\frac{p}{2}, \quad \frac{p}{2}-1, \ldots, \quad 1-\frac{p}{2}, \quad-\frac{p}{2}
$$

and corresponding eigenvectors are given by

$$
\left|J_{3} ; \frac{p}{2}\right\rangle, \quad J_{-}\left|J_{3} ; \frac{p}{2}\right\rangle, \ldots, \quad J_{-}^{p}\left|J_{3} ; \frac{p}{2}\right\rangle
$$

where $\left|J_{3} ; \frac{p}{2}\right\rangle$ is a normalized eigenvector with eigenvalue $p / 2$ and $J_{-}=J_{1}-$ $i J_{2}$. Let $j=p / 2$ and $\left|J_{3} ; m\right\rangle=J_{-}^{j-m}\left|J_{3} ; j\right\rangle / \| J_{-}^{j-m}\left|J_{3} ; j\right\rangle \|$. It is shown that

$$
\left\langle J_{3} ; m^{\prime}\left|J_{-}\right| J_{3} ; m\right\rangle=\delta_{m^{\prime} m-1} \sqrt{(j+m)(j-m+1)}
$$

We consider the nonstandard version of this representation; let $p$ be an infinite hypernatural number, and $J_{k}$ be the internal matrices satisfying the above commutation relations. Define $Q$ and $P$ by $Q=J_{1} / \sqrt{j}, P=J_{2} / \sqrt{j}$.

Theorem 3.1: Let $S \subset{ }^{\star} \mathbf{C}^{\nu}$ be the subspace finitely spanned by $\mid J_{3} ; j-$ $k\rangle, \quad(k=0,1, \ldots<\infty)$, i.e.,

$$
S=\left\{\sum_{k=0}^{n} c_{k}\left|J_{3} ; j-k\right\rangle: c_{k} \in{ }^{\star} \mathbf{C},\left|c_{k}\right|<\infty, n \in \mathbf{N}\right\}
$$

$S$ is an invariant subspace of $P$ and $Q$, and

$$
[Q, P]|\xi\rangle \approx i|\xi\rangle
$$

for each $|\xi\rangle \in S$, and hence $(Q, P, S)$ is a representation of CCR.

Proof: The invariance of $S$ is seen from

$$
\begin{gathered}
Q\left|J_{3} ; j-k\right\rangle=\frac{1}{\sqrt{j}} \frac{1}{2}\left(J_{-}+J_{-}^{\dagger}\right)\left|J_{3} ; j-k\right\rangle \\
=\frac{1}{2} \sqrt{(2-k / j)(k+1)}\left|J_{3} ; j-k-1\right\rangle+\frac{1}{2} \sqrt{k(2-k / j+1 / j)}\left|J_{3} ; j-k+1\right\rangle \in S \\
P\left|J_{3} ; j-k\right\rangle=\frac{1}{\sqrt{j}} \frac{i}{2}\left(J_{-}^{\dagger}-J_{-}\right)\left|J_{3} ; j-k\right\rangle \\
=\frac{i}{2} \sqrt{(2-k / j)(k+1)}\left|J_{3} ; j-k-1\right\rangle-\frac{i}{2} \sqrt{k(2-k / j+1 / j)}\left|J_{3} ; j-k+1\right\rangle \in S
\end{gathered}
$$

And we have $[Q, P]\left|J_{3} ; j-k\right\rangle=\frac{i}{j} J_{3}\left|J_{3} ; j-k\right\rangle=i(1-k / j)\left|J_{3} ; j-k\right\rangle \approx$ $i\left|J_{3} ; j-k\right\rangle$. Q.E.D.

We find the rotation covariance of these operators;

$$
Q \cos \theta+P \sin \theta=e^{i \theta J_{3}} Q e^{-i \theta J_{3}} .
$$


Moreover, it turns out that this representation is convenient in dealing with coherent states. Let $R(\theta, \phi)$ denote the rotation matrix

$$
R(\theta, \phi)=e^{i \theta\left(J_{1} \sin \phi-J_{2} \cos \phi\right)}
$$

$R(\theta, \phi)$ has also the expression ${ }^{11}$

$$
R(\theta, \phi)=e^{\mu J_{-}} e^{-\log \left(1+|\mu|^{2}\right) J_{3}} e^{-\mu^{*} J_{-}^{\dagger}}
$$

where $\mu=e^{i \phi} \tan \frac{\theta}{2}$. Define $|\theta, \phi\rangle$ by $|\theta, \phi\rangle=R(\theta, \phi)\left|J_{3} ; j\right\rangle$. It is shown that

$$
|\theta, \phi\rangle=\frac{1}{\left(1+|\mu|^{2}\right)^{j}} \sum_{k=0}^{2 j}\left(\begin{array}{c}
2 j \\
k
\end{array}\right)^{1 / 2} \mu^{k}\left|J_{3} ; j-k\right\rangle
$$

If $|\mu| \sqrt{j}<\infty$ and $k<\infty$,

$$
\frac{1}{\left(1+|\mu|^{2}\right)^{j}} \approx e^{-j|\mu|^{2}}, \quad\left(\begin{array}{c}
2 j \\
k
\end{array}\right)^{1 / 2} \mu^{k} \approx \frac{(\sqrt{2 j} \mu)^{k}}{\sqrt{k !}}
$$

Thus we find

$$
\left\langle J_{3} ; j-k \mid \theta, \phi\right\rangle \approx e^{-|z|^{2} / 2} \frac{z^{k}}{k !}
$$

where $z=\mu \sqrt{2 j}$. Corresponding standard coherent state $|z\rangle$ satisfies

$$
\langle k \mid z\rangle=e^{-|z|^{2} / 2} \frac{z^{k}}{k !}
$$

where $|k\rangle=a^{\dagger k}|0\rangle / \| a^{\dagger k}|0\rangle \|, a^{\dagger}$ being the standard bose creation operator and $|0\rangle$ the vacuum state.

\section{REPRESENTATIONS OF $S O(n)$}

The hyperfinite-dimensional representation of CCR which has more general rotation covariance is constructed by hyperfinite-dimensional representation of $s o(n)$. This section reviews the method of spin representation in use of Clifford algebra. Let $C\left(\mathbf{R}_{-}^{n}\right)$ be the $\mathbf{R}$-vector space the base of which is the $2^{n}$ elements

$$
\begin{gathered}
1, e_{1}, \ldots, e_{n}, e_{1} e_{2}, \ldots, e_{i} e_{j}(i<j), \ldots, e_{n-1} e_{n}, \ldots \\
e_{i_{1}} \cdots e_{i_{k}}\left(i_{1}<\cdots<i_{k}\right), \ldots, e_{1} \cdots e_{n}
\end{gathered}
$$

Define the product of these elements by

$$
\left(e_{i_{1}} \cdots e_{i_{k}}\right)\left(e_{j_{1}} \cdots e_{j_{l}}\right)=e_{i_{1}} \cdots e_{i_{k}} e_{j_{1}} \cdots e_{j_{l}}
$$


and require the relation

$$
e_{i}^{2}=-1, \quad(i=1, \ldots, n), \quad e_{i} e_{j}=-e_{j} e_{i}(i \neq j), \quad(\lambda 1) e_{i}=e_{i}(\lambda 1) \quad(\lambda \in \mathbf{R})
$$

Assume that 1 is the unit of the product, and that the product is associative. Now, $C\left(\mathbf{R}_{-}^{n}\right)$ is $2^{n}$-dimensional $\mathbf{R}$-algebra. $C\left(\mathbf{R}_{-}^{n}\right)$ is called Clifford algebra.

Let us identify $\left(\lambda_{1}, \ldots, \lambda_{n}\right) \in \mathbf{R}^{n}$ with $\sum_{k=1}^{n} \lambda_{k} e_{k}$. Then we have $S^{n-1} \subset$ $\mathbf{R}^{n} \subset C\left(\mathbf{R}_{-}^{n}\right)\left(S^{n-1}\right.$ is the unit sphere in $\left.\mathbf{R}^{n}\right)$. The following fact is shown.

Theorem 4.1: $\operatorname{Spin}(n)$ defined by

$$
\operatorname{Spin}(n)=\left\{\alpha \in C\left(\mathbf{R}_{-}^{n}\right) \mid \alpha=a_{1} \cdots a_{m}, a_{i} \in S^{n-1}, m=2,4, \ldots\right\}
$$

is a topological group, and isomorphic to the covering group of $S O(n) . e_{i j}=$ $e_{i} e_{j}(i, j=1, \ldots, n)$ is a base (of a representation) of $s o(n)$.

If $n=2 \nu+1, e_{1}, \ldots, e_{n}$ is represented by $2^{\nu} \times 2^{\nu}$ matrices as follows. Let $V_{k} \simeq \mathbf{C}^{2}(k=1, \ldots, \nu)$. Pauli matrices $\sigma_{1, k}, \sigma_{2, k}, \sigma_{3, k}$ that act on $V_{k}$ are represented as

$$
\sigma_{1, k}=\left(\begin{array}{cc}
0 & 1 \\
1 & 0
\end{array}\right), \quad \sigma_{2, k}=\left(\begin{array}{cc}
0 & -i \\
i & 0
\end{array}\right), \quad \sigma_{3, k}=\left(\begin{array}{cc}
1 & 0 \\
0 & -1
\end{array}\right) .
$$

Define $V$ by

$$
V=V_{1} \otimes \cdots \otimes V_{\nu}
$$

and define $\hat{\sigma}_{c, k}$ and $\gamma_{i}$ that acts on $V$ by

$$
\begin{gathered}
\hat{\sigma}_{c, k}=\overbrace{1 \otimes \cdots \otimes 1}^{k-1} \otimes \sigma_{c, k} \otimes 1 \otimes \cdots \otimes 1, \quad c=1,2,3 \\
\gamma_{2 k-1}=\hat{\sigma}_{2, k} \hat{\sigma}_{3, k+1} \cdots \hat{\sigma}_{3, \nu} \\
\gamma_{2 k}=-\hat{\sigma}_{1, k} \hat{\sigma}_{3, k+1} \cdots \hat{\sigma}_{3, \nu} \\
\gamma_{2 \nu+1}=\hat{\sigma}_{3,1} \hat{\sigma}_{3,2} \cdots \hat{\sigma}_{3, \nu}
\end{gathered}
$$

Direct calculations show the following relations.

$$
\left\{\gamma_{i}, \gamma_{j}\right\}=2 \delta_{i j}, \quad i, j=1, \ldots, 2 \nu
$$

Thus, we can take $e_{j}=i \gamma_{j}$ (this is called the spin representation).

Moreover we have the tensor product representation of $s o(n)$ from one constructed above;

$$
\tilde{e}_{i j}=\sum_{l=0}^{p-1} \overbrace{1 \otimes \cdots \otimes 1}^{l} \otimes e_{i j} \otimes \overbrace{1 \otimes \cdots \otimes 1}^{p-l-1}
$$

also genarate a representation of $s o(n)$.

In the next section, we introduce a hyperfinite representation of so(n), using these mathematical tools. 


\section{HYPERFINITE PARAFERMI REPRE- SENTATION}

In this section, we construct a hyperfinite-dimensional representation of the algebra of standard infinite bose oscillators, that is, the algebra generated by bose annihilation operators $a_{1}, a_{2}, \ldots$ satisfying $\left[a_{j}, a_{k}^{\dagger}\right]=\delta_{j k}$ and $\left[a_{j}, a_{k}\right]=0$, with the condition that nonzero vector $|0\rangle$ that satisfies $a_{k}|0\rangle=0$ is unique except the scalar multiples (the uniqueness of vacuum). This representation is constructed by using hyperfinite internal representations of the algebra of parafermi oscillators, which is mathematically equivalent to the hyperfinitedimensional spin representation of $s o(n)$.

Definition 5.1:12,13 Let $\nu \in \mathbf{N}$, and suppose that for some $d \in \mathbf{N}$, $b_{1}, \ldots, b_{\nu} \in M(d, \mathbf{C})$ (i.e., $b_{1}, \ldots, b_{\nu}$ are finite-dimensional matrices). $b_{1}, \ldots, b_{\nu}$ is called annihilation operators of parafermi oscillators of order $p \in \mathbf{N}$ if they satisfy

$$
\begin{aligned}
{\left[b_{k},\left[b_{l}^{\dagger}, b_{m}\right]\right] } & =2 \delta_{k l} b_{m} \\
{\left[b_{k},\left[b_{l}^{\dagger}, b_{m}^{\dagger}\right]\right] } & =2 \delta_{k l} b_{m}^{\dagger}-2 \delta_{k m} b_{l}^{\dagger} \\
{\left[b_{k},\left[b_{l}, b_{m}\right]\right] } & =0 .
\end{aligned}
$$

and the uniqueness of vacuum $|0\rangle$, and,

$$
b_{k} b_{l}^{\dagger}|0\rangle=\delta_{k l} p|0\rangle \text {. }
$$

$b_{1}^{\dagger}, \ldots, b_{\nu}^{\dagger}$ are called creation operators of parafermi oscillators of order $p . H y$ perfinite annihilation operators of parafermi oscillators are the internal matrices defined in the above definition by substituting ${ }^{\star} \mathbf{N}$ and ${ }^{\star} \mathbf{C}$ for $\mathbf{N}$ and C, respectively.

Green $^{12}$ has given a class of representations of the above commutation relations of parafermi creation and annihilation operators. In the so-called Green representation for the cases of order $p$, the parafermi operators $b_{k}$ are expressed by the form

$$
b_{k}=\sum_{\alpha=1}^{p} b_{k}^{(\alpha)},
$$

where the Green-component operators $b_{k}^{(\alpha)}$ satisfy the commutation relations

$$
\begin{gathered}
\left\{b_{k}^{(\alpha)}, b_{l}^{(\alpha)^{\dagger}}\right\}=\delta_{k l}, \quad\left\{b_{k}^{(\alpha)}, b_{l}^{(\alpha)}\right\}=0, \\
{\left[b_{k}^{(\alpha)}, b_{l}^{(\beta)^{\dagger}}\right]=\left[b_{k}^{(\alpha)}, b_{l}^{(\beta)}\right]=0, \quad(\alpha \neq \beta)}
\end{gathered}
$$

where $\{A, B\}=A B+B A$, and the uniqueness of vacuum $|0\rangle$ such that

$$
b_{k}^{(\alpha)}|0\rangle=0 \quad \text { for all } k, \alpha .
$$


Green representation is essentially equivalent to the tensor product representation of Clifford algebra representation of $s o(2 \nu)$. In fact, we easily verify that $e_{1}, \ldots, e_{2 \nu}$ defined by $e_{2 k-1}=i\left(b_{k}^{\dagger}+b_{k}\right), e_{2 k}=b_{k}^{\dagger}-b_{k}$ form the generator of a Clifford algebra (i.e., $e_{i}^{2}=-1$ and $e_{i} e_{j}=-e_{j} e_{i}(i \neq j)$ hold). Thus, we can construct a $2^{p \nu}$-dimensional representation of Green components by using a spin representation of the Clifford algebra as follows. Let $V_{k}^{(\alpha)} \simeq \mathbf{C}^{2}(k=1, \ldots, \nu, \alpha=1, \ldots, p)$. Pauli matrices $\sigma_{1, k}^{(\alpha)}, \sigma_{2, k}^{(\alpha)}$ and $\sigma_{3, k}^{(\alpha)}$ that act on $V_{k}^{(\alpha)}$ are represented as

$$
\sigma_{1, k}^{(\alpha)}=\left(\begin{array}{cc}
0 & 1 \\
1 & 0
\end{array}\right), \quad \sigma_{2, k}^{(\alpha)}=\left(\begin{array}{cc}
0 & -i \\
i & 0
\end{array}\right), \quad \sigma_{3, k}^{(\alpha)}=\left(\begin{array}{cc}
1 & 0 \\
0 & -1
\end{array}\right) .
$$

Define $V^{(\alpha)}$ by

$$
V^{(\alpha)}=V_{1}^{(\alpha)} \otimes \cdots \otimes V_{\nu}^{(\alpha)},
$$

and define $\hat{\sigma}_{c, k}^{(\alpha)}$ and $\gamma_{i}^{(\alpha)}(k=1, \ldots, \nu, i=1, \ldots, 2 \nu)$ that acts on $V^{(\alpha)}$ by

$$
\begin{gathered}
\hat{\sigma}_{c, k}^{(\alpha)}=\overbrace{1 \otimes \cdots \otimes 1}^{k-1} \otimes \sigma_{c, k}^{(\alpha)} \otimes 1 \otimes \cdots \otimes 1, \quad c=1,2,3, \\
\gamma_{2 k-1}^{(\alpha)}=\hat{\sigma}_{2, k}^{(\alpha)} \hat{\sigma}_{3, k+1}^{(\alpha)} \cdots \hat{\sigma}_{3, \nu}^{(\alpha)}, \\
\gamma_{2 k}^{(\alpha)}=-\hat{\sigma}_{1, k}^{(\alpha)} \hat{\sigma}_{3, k+1}^{(\alpha)} \cdots \hat{\sigma}_{3, \nu}^{(\alpha)} .
\end{gathered}
$$

Operators $b_{k}^{(\alpha)}(k=1, \ldots, \nu)$ defined by

$$
b_{k}^{(\alpha)}=\frac{1}{2}\left(\gamma_{2 k-1}^{(\alpha)}-i \gamma_{2 k}^{(\alpha)}\right)
$$

satisfy the relations

$$
\left\{b_{k}^{(\alpha)}, b_{l}^{(\alpha) \dagger}\right\}=\delta_{k l}, \quad\left\{b_{k}^{(\alpha)}, b_{l}^{(\alpha)}\right\}=0,
$$

for all $k, l=1, \ldots, \nu$.

Define $V$ and $\tilde{b}_{k}^{(\alpha)}(k=1, \ldots, \nu)$ acting on $V$ by

$$
\begin{gathered}
V=V^{(1)} \otimes \cdots \otimes V^{(p)}, \\
\tilde{b}_{k}^{(\alpha)}=\overbrace{1 \otimes \cdots \otimes 1}^{\alpha-1} \otimes b_{k}^{(\alpha)} \otimes 1 \otimes \cdots \otimes 1 .
\end{gathered}
$$

We see that for all $k, l=1, \ldots, \nu$,

$$
\begin{gathered}
\left\{\tilde{b}_{k}^{(\alpha)}, \tilde{b}_{l}^{(\alpha) \dagger}\right\}=\delta_{k l}, \quad\left\{\tilde{b}_{k}^{(\alpha)}, \tilde{b}_{l}^{(\alpha)}\right\}=0, \\
{\left[\tilde{b}_{k}^{(\alpha)}, \tilde{b}_{l}^{(\beta) \dagger}\right]=\left[\tilde{b}_{k}^{(\alpha)}, \tilde{b}_{l}^{(\beta)}\right]=0, \quad(\alpha \neq \beta) .}
\end{gathered}
$$


Let $|0\rangle_{k}^{(\alpha)} \in V_{k}^{(\alpha)}$ denote the normalized vector such that $b_{k}^{(\alpha)}|0\rangle_{k}^{(\alpha)}=0$. Define $|0\rangle^{(\alpha)}$ and $|0\rangle$ by

$$
\begin{gathered}
|0\rangle^{(\alpha)}=|0\rangle_{1}^{(\alpha)} \otimes \cdots \otimes|0\rangle_{\nu}^{(\alpha)}, \\
|0\rangle=|0\rangle^{(\alpha)} \otimes \cdots \otimes|0\rangle^{(p)} .
\end{gathered}
$$

Now, we find that $\tilde{b}_{1}^{(\alpha)}, \ldots, \tilde{b}_{\nu}^{(\alpha)}$ are $2^{p \nu}$-dimensional representations of Green components and $|0\rangle$ is the vacuum. Thus, $b_{k}=\sum_{\alpha=1}^{p} \tilde{b}_{k}^{(\alpha)},(k=1, \ldots, \nu)$ are $2^{p \nu}$-dimensional representations of annihilation operators of $\nu$ parafermi oscillators of order $p$. Let us call the above representation of the algebra of parafermi oscillators spin representation.

Define $\sigma_{ \pm, k}^{(\alpha)}$ by

$$
\sigma_{ \pm, k}^{(\alpha)}=\left(\sigma_{1, k}^{(\alpha)} \pm i \sigma_{2, k}^{(\alpha)}\right) / 2
$$

and $|1\rangle_{k}^{(\alpha)} \in V_{k}^{(\alpha)}$ by

$$
\begin{gathered}
|1\rangle_{k}^{(\alpha)}=\sigma_{+, k}^{(\alpha)}|0\rangle_{k}^{(\alpha)} \\
\left\{\left(\left|e_{1}^{(1)}\right\rangle_{1}^{(1)} \cdots\left|e_{\nu}^{(1)}\right\rangle_{\nu}^{(1)}\right) \cdots\left(\left|e_{1}^{(p)}\right\rangle_{1}^{(p)} \cdots\left|e_{\nu}^{(p)}\right\rangle_{\nu}^{(p)}\right): e_{k}^{(\alpha)}=0,1\right\}
\end{gathered}
$$

( $\otimes$ 's are omitted) is a complete orthonormal system of $V$. We write the vectors simply as $\left|\left\{e_{k}^{(\alpha)}\right\}\right\rangle$.

Number operator $N$ on $V$ and the related operators $N_{k}, N^{(\alpha)}$ are defined as follows:

$$
\begin{gathered}
N_{k}^{(\alpha)}=\frac{1}{2}\left(1+\sigma_{3, k}^{(\alpha)}\right)=\left(\begin{array}{cc}
1 & 0 \\
0 & 0
\end{array}\right), \\
\hat{N}_{k}^{(\alpha)}=\overbrace{1 \otimes \cdots \otimes 1}^{k-1} \otimes N_{k}^{(\alpha)} \otimes \overbrace{1 \otimes \cdots \otimes 1}^{\nu-k}, \\
\tilde{N}_{k}^{(\alpha)}=\overbrace{1 \otimes \cdots \otimes 1}^{\alpha-1} \otimes \hat{N}_{k}^{(\alpha)} \otimes \overbrace{1 \otimes \cdots \otimes 1}^{p-\alpha}, \\
N_{k}=\sum_{\alpha=1}^{p} \tilde{N}_{k}^{(\alpha)}, \quad N^{(\alpha)}=\sum_{k=1}^{\nu} \tilde{N}_{k}^{(\alpha)}, \quad N=\sum_{\alpha=1}^{p} N^{(\alpha)},
\end{gathered}
$$

We see that

$$
N\left|\left\{e_{k}^{(\alpha)}\right\}\right\rangle=n\left|\left\{e_{k}^{(\alpha)}\right\}\right\rangle
$$

where $n$ is the number of $e_{k}^{(\alpha)}$ 's that is equal to 1 . It is easily shown that

$$
\begin{aligned}
& \tilde{b}_{k}^{(\alpha) \dagger} \tilde{b}_{k}^{(\alpha)}=\tilde{N}_{k}^{(\alpha)}, \quad \tilde{b}_{k}^{(\alpha)} \tilde{b}_{k}^{(\alpha) \dagger}=1-\tilde{N}_{k}^{(\alpha)}, \quad N_{k}=\frac{1}{2}\left(\left[b_{k}^{\dagger}, b_{k}\right]+p\right), \\
& {\left[N_{k}, N_{l}\right]=0, \quad N_{k} b_{k}=b_{k}\left(N_{k}-1\right), \quad N_{k} b_{k}^{\dagger}=b_{k}^{\dagger}\left(N_{k}+1\right), \text { etc. }}
\end{aligned}
$$

Lemma 5.1: Suppose that hyperfinite parafermi annihilation operators $b_{1}, \ldots, b_{\nu}$ are represented by spin representation, and that their order $p$ is an infinite hypernatural number $\left(b_{1}, \ldots, b_{\nu}\right.$ are $2^{p \nu} \times 2^{p \nu}$ internal matrices acting on $\left.{ }^{\star} \mathbf{C}^{2^{p \nu}}\right)$. If $|\xi\rangle \in{ }^{\star} \mathbf{C}^{2^{p \nu}}$ satisfies $\langle\xi \mid \xi\rangle,\left\langle\xi\left|N^{2}\right| \xi\right\rangle<\infty$, and $k \neq l(k, l=$ $1,2, \ldots, \nu)$, then 
(i) $\left[\beta_{k}, \beta_{l}\right]|\xi\rangle \approx\left[\beta_{k}, \beta_{l}^{\dagger}\right]|\xi\rangle \approx 0$

(ii) $\left[\beta_{k}, \beta_{k}^{\dagger}\right]|\xi\rangle \approx|\xi\rangle$.

(iii) $\beta_{k} \beta_{k}^{\dagger n}|\xi\rangle \approx\left(\beta_{k}^{\dagger n} \beta_{k}+n \beta_{k}^{\dagger n-1}\right)|\xi\rangle$.

where $\beta_{k}=p^{-1 / 2} b_{k}$ (normalization of $b_{k}$ ) and $n<\infty$.

Proof: (i) Notice that

$$
\left[\beta_{k}, \beta_{l}\right]=\frac{2}{p} \sum_{\alpha=1}^{p} \tilde{b}_{k}^{(\alpha)} \tilde{b}_{l}^{(\alpha)}, \quad\left[\beta_{k}, \beta_{l}^{\dagger}\right]=\frac{2}{p} \sum_{\alpha=1}^{p} \tilde{b}_{k}^{(\alpha)} \tilde{b}_{l}^{(\alpha) \dagger} .
$$

Direct calculations show that

$$
\begin{aligned}
& b_{k}^{(\alpha)} b_{l}^{(\alpha)}= \begin{cases}-\hat{\sigma}_{3, k+1}^{(\alpha)} \cdots \hat{\sigma}_{3, l}^{(\alpha)} \hat{\sigma}_{-, k}^{(\alpha)} \hat{\sigma}_{-, l}^{(\alpha)} & (l>k) \\
-\hat{\sigma}_{3, l+1}^{(\alpha)} \cdots \hat{\sigma}_{3, k}^{(\alpha)} \hat{\sigma}_{-, k}^{(\alpha)} \hat{\sigma}_{-, l}^{(\alpha)} & (k>l)\end{cases} \\
& b_{k}^{(\alpha)} b_{l}^{(\alpha)^{\dagger}}= \begin{cases}\hat{\sigma}_{3, k}^{(\alpha)} \cdots \hat{\sigma}_{3, l}^{(\alpha)} \hat{\sigma}_{+, k}^{(\alpha)} \hat{\sigma}_{-, l}^{(\alpha)} & (l>k) \\
\hat{\sigma}_{3, l+1}^{(\alpha)} \cdots \hat{\sigma}_{3, k}^{(\alpha)} \hat{\sigma}_{+, k}^{(\alpha)} \hat{\sigma}_{-, l}^{(\alpha)} & (k>l)\end{cases}
\end{aligned}
$$

Hence, using $N_{k}^{(\alpha)}+N_{l}^{(\alpha)}-2 N_{k}^{(\alpha)} N_{l}^{(\alpha)}=\left(N_{k}^{(\alpha)}-N_{l}^{(\alpha)}\right)^{2} \geq 0$,

$$
\begin{aligned}
\| \tilde{b}_{k}^{(\alpha)} \tilde{b}_{l}^{(\alpha)}|\xi\rangle \|^{2} & =\left\langle\xi\left|\tilde{\sigma}_{+, k}^{(\alpha)} \tilde{\sigma}_{+, l}^{(\alpha)} \tilde{\sigma}_{-, l}^{(\alpha)} \tilde{\sigma}_{-, k}^{(\alpha)}\right| \xi\right\rangle \\
& =\left\langle\xi\left|\tilde{N}_{k}^{(\alpha)} \tilde{N}_{l}^{(\alpha)}\right| \xi\right\rangle \\
& \leq \frac{1}{2}\left\langle\xi\left|\tilde{N}_{k}^{(\alpha)}+\tilde{N}_{l}^{(\alpha)}\right| \xi\right\rangle \\
& \leq \frac{1}{2}\left\langle\xi\left|\tilde{N}^{(\alpha)}\right| \xi\right\rangle .
\end{aligned}
$$

Thus,

$$
\begin{aligned}
\|\left[\beta_{k}, \beta_{l}\right]|\xi\rangle \| & =\| \frac{2}{p} \sum_{\alpha=1}^{p} \tilde{b}_{k}^{(\alpha)} \tilde{b}_{l}^{(\alpha)}|\xi\rangle \| \\
& \leq \frac{2}{p} \sum_{\alpha=1}^{p} \| \tilde{b}_{k}^{(\alpha)} \tilde{b}_{l}^{(\alpha)}|\xi\rangle \| \\
& \leq \frac{2}{p} \sum_{\alpha=1}^{p} \sqrt{\frac{1}{2}\left\langle\xi\left|\tilde{N}^{(\alpha)}\right| \xi\right\rangle} \approx 0 .
\end{aligned}
$$

The last equation is seen from $\sum_{\alpha=1}^{p}\left\langle\xi\left|N^{(\alpha)}\right| \xi\right\rangle=\langle\xi|N| \xi\rangle<\infty$; under this condition we find that the number of $\alpha$ 's such that $\left\langle\xi\left|N^{(\alpha)}\right| \xi\right\rangle \not \approx 0$ is finite.

In a similar way, it is shown that

$$
\|\left[\beta_{k}, \beta_{l}^{\dagger}\right]|\xi\rangle\left\|\leq \frac{2}{p} \sum_{\alpha=1}^{p}\right\| \tilde{b}_{k}^{(\alpha)} \tilde{b}_{l}^{(\alpha) \dagger}|\xi\rangle \|
$$




$$
\begin{aligned}
& \leq \frac{2}{p} \sum_{\alpha=1}^{p} \sqrt{\left\langle\xi\left|\tilde{N}_{l}^{(\alpha)}\left(1-\tilde{N}_{k}^{(\alpha)}\right)\right| \xi\right\rangle} \\
& \leq \frac{2}{p} \sum_{\alpha=1}^{p} \sqrt{\left\langle\xi\left|\tilde{N}_{l}^{(\alpha)}\right| \xi\right\rangle} \approx 0 .
\end{aligned}
$$

(ii) A direct calculation shows that

$$
\begin{gathered}
{\left[\beta_{k}, \beta_{k}^{\dagger}\right]=-\frac{1}{p} \sum_{\alpha=1}^{p} \tilde{\sigma}_{3, k}^{(\alpha)},} \\
\left(\left[\beta_{k}, \beta_{k}^{\dagger}\right]-1\right)^{2}=\frac{4}{p^{2}}\left(\sum_{\alpha=1}^{p} N_{k}^{(\alpha)}\right)^{2} .
\end{gathered}
$$

Hence,

$$
\begin{aligned}
\|\left[\beta_{k}, \beta_{k}^{\dagger}\right]|\xi\rangle-|\xi\rangle \|^{2}= & \frac{4}{p^{2}}\left\langle\xi\left|\left(\sum_{\alpha=1}^{p} N_{k}^{(\alpha)}\right)\right| \xi\right\rangle . \\
& \leq \frac{4}{p^{2}}\left\langle\xi\left|N^{2}\right| \xi\right\rangle \approx 0 .
\end{aligned}
$$

(iii) is shown by the induction for $n$, using (ii). Q.E.D.

Suppose the number of parafermi oscillators $\nu$ and their order $p$ are infinite hypernatural numbers. When $n_{i}$ is nonnegative integer for any $i=1,2, \ldots<$ $\infty$, and the number of $n_{i}$ 's such that $n_{i} \neq 0$ is finite, we will define $\left|n_{1}, n_{2}, \ldots\right\rangle$ by

$$
\left|n_{1}, n_{2}, \ldots\right\rangle=\frac{b_{1}^{\dagger n_{1}} b_{2}^{\dagger n_{2}} \cdots|0\rangle}{\| b_{1}^{\dagger n_{1}} b_{2}^{\dagger n_{2}} \cdots|0\rangle \|} .
$$

$b_{1}^{\dagger n_{1}} b_{2}^{\dagger n_{2}} \ldots$ is the product of a finite number of operators, so it is well-defined. $N_{k}\left|n_{1}, n_{2}, \ldots\right\rangle=n_{k}\left|n_{1}, n_{2}, \ldots\right\rangle$ is easily shown, and hence, since $N_{k}$ is hermitian, the set of the vectors of the form $\left|n_{1}, n_{2}, \ldots\right\rangle$ is an orthonormal system.

Lemma 5.2:

(i) $\beta_{k}^{\dagger} \beta_{k}\left|n_{1}, n_{2}, \ldots\right\rangle \approx n_{k}\left|n_{1}, n_{2}, \ldots\right\rangle$,

(ii) $\beta_{k} \beta_{k}^{\dagger}\left|n_{1}, n_{2}, \ldots\right\rangle \approx\left(n_{k}+1\right)\left|n_{1}, n_{2}, \ldots\right\rangle$,

(iii) $\| \beta_{1}^{\dagger n_{1}} \beta_{2}^{\dagger n_{2}} \cdots|0\rangle \| \approx \sqrt{n_{1} ! n_{2} ! \cdots}$,

(iv) $\beta_{k}^{\dagger}\left|n_{1}, n_{2}, \ldots\right\rangle \approx \sqrt{n_{k}+1}\left|n_{1}, n_{2}, \ldots, n_{k}+1, \ldots\right\rangle$,

(v) $\beta_{k}\left|n_{1}, n_{2}, \ldots\right\rangle \approx \sqrt{n_{k}}\left|n_{1}, n_{2}, \ldots, n_{k}-1, \ldots\right\rangle$.

Proof: (i) and (ii) are shown by Lemma 5.1(i)-(iii). (iii) is shown by the induction for $n_{1}, n_{2}, \ldots$, using (ii) and Lemma 5.1(i). (iv) and (v) are shown by (iii) and Lemma 5.1(i)(iii). Q.E.D. 
Define a set $D \subset{ }^{\star} \mathbf{C}^{2^{p \nu}}$ by

$$
D=\left\{\frac{\beta_{k_{1}}^{\dagger} \cdots \beta_{k_{n}}^{\dagger}|0\rangle}{\| \beta_{k_{1}}^{\dagger} \cdots \beta_{k_{n}}^{\dagger}|0\rangle \|} \mid n, k_{1}, \ldots, k_{n} \in \mathbf{N}\right\} \cup\{|0\rangle\} .
$$

Clearly, every vector in $D$ is a normalized eigenvector of the number operator $N$ with a finite eigenvalue. Let $S$ denote the external subspace of ${ }^{\star} \mathbf{C}^{2^{p \nu}}$ spanned by $D$, i.e.,

$$
S=\left\{\sum_{i=1}^{n} c_{i}|\xi\rangle: \quad c_{i} \in{ }^{\star} \mathbf{C},\left|c_{i}\right|<\infty, n \in \mathbf{N},|\xi\rangle \in D\right\} .
$$

The following theorem follows from Lemma 5.1 and 5.2.

Theorem 5.3: $\left(\beta_{k}, S\right)(k \in \mathbf{N})$ is a representation of CCR of countablyinfinite degree of freedom, i.e., $S$ is invariant in respect to $\beta_{k}$ and $\beta_{k}^{\dagger}$ for every $k \in \mathbf{N}$, and

$$
\begin{gathered}
{\left[\beta_{k}, \beta_{l}\right]|\xi\rangle \approx 0} \\
{\left[\beta_{k}, \beta_{l}^{\dagger}\right]|\xi\rangle \approx \delta_{k l}|\xi\rangle}
\end{gathered}
$$

for any $|\xi\rangle \in S$. Moreover, the uniqueness of vacuum is satisfied in the following sense: if $|\xi\rangle \in S,\langle\xi \mid \xi\rangle=1$ and $\beta_{k}|\xi\rangle \approx 0$ for all $k \in \mathbf{N}$, then $|\langle\xi \mid 0\rangle| \approx 1$.

\section{APPENDIX: NONSTANDARD ANALYSIS}

This section briefly outlines the basic notions of nonstandard analysis along Hurd and Loeb ${ }^{6}$, and reviews some development in the field of hyperfinitedimensional linear space theory. Let $X$ be a set and $\mathcal{P}(X)$ the power set of $X$, that is, the set of all subsets of $X$. The superstructure over $X$, denoted by $V(X)$, is defined by the following recursion:

$$
\begin{gathered}
V_{0}(X)=X, V_{n+1}(X)=V_{n}(X) \cup \mathcal{P}\left(V_{n}(X)\right), \\
V(X)=\bigcup_{n \in \mathbf{N}} V_{n}(X) .
\end{gathered}
$$

Let us regard any element of $X$ as a nonset here; hence $x \in V(X)$ is a set iff $x \in V(X) \backslash X$. Let $\mathbf{C}$ be the set of complex numbers. $V(\mathbf{C})$ contains all the structures that we usually use in quantum physics, for instance, separable Hilbert space $\mathcal{H}$.

$V(X)$ is called a nonstandard extension of $V(\mathbf{C})$ if there exists a map $\star: V(\mathbf{C}) \longrightarrow V(X)$ satisfying the following conditions:

$$
(1) \star \text { is an injective mapping from } V(\mathbf{C}) \text { to } V(X) \text {, }
$$


$(2){ }^{\star} \mathbf{C}=X$,

(3) (Transfer Principle) Let $\phi$ be a sentence in terms of $V(\mathbf{C})$, and ${ }^{\star} \phi$ the sentence "transfered" from $\phi$ by mapping $\star . \phi$ is true iff ${ }^{\star} \phi$ is true.

Transfer Principle needs more explanation. Although the exact description of it needs more definitions of some notions in mathematical logic, one can find the intuitive meaning of it without them, in an example. A sentence in terms of $V(\mathbf{C})$ is constructed from the symbols for logical connectives $\neg, \wedge, \vee, \Rightarrow, \Leftrightarrow$, quantifiers $\forall, \exists$, individual variables $x, y, z, \ldots$, two predicates $=, \in$, parentheses $($,$) , and elements of V(\mathbf{C})$. We will consider an example. Let $\mathbf{R}$ denote the set of real numbers. Define $G_{<} \in V(\mathbf{C})$ by $G_{<}=\{(x, y) \mid x, y \in \mathbf{R}, x<y\}$, where $(x, y)$ is identified as $\{\{x\}, y\}$.

$$
\begin{gathered}
(\forall x)(\forall y)\left(x \in \mathbf{R} \wedge y \in \mathbf{R} \wedge(x, y) \in G_{<} \Rightarrow(\exists z)(z \in \mathbf{R}\right. \\
\left.\left.\wedge(x, z) \in G_{<} \wedge(z, y) \in G_{<}\right)\right)
\end{gathered}
$$

is a sentence in terms of $V(\mathbf{C})$ because $\mathbf{R}, G_{<} \in V(\mathbf{C})$. Let $\phi$ denote this sentence. $\phi$ means that $\mathbf{R}$ is dense, and hence $\phi$ is true. The "transfered" sentence ${ }^{\star} \phi$ is as follows:

$$
\begin{gathered}
(\forall x)(\forall y)\left(x \in { } ^ { \star } \mathbf { R } \wedge y \in { } ^ { \star } \mathbf { R } \wedge ( x , y ) \in { } ^ { \star } G _ { < } \Rightarrow ( \exists z ) \left(z \in{ }^{\star} \mathbf{R}\right.\right. \\
\left.\left.\wedge(x, z) \in{ }^{\star} G_{<} \wedge(z, y) \in{ }^{\star} G_{<}\right)\right)
\end{gathered}
$$

By Transfer Principle, ${ }^{\star} \phi$ is true $\left({ }^{\star} \mathbf{R}\right.$ is called $\star$-dense $)$.

$u \in V(X)$ is called standard if there is $x \in V(\mathbf{C})$ such that $u={ }^{\star} x$, and called internal if there is $x \in V(\mathbf{C})$ such that $u \in{ }^{\star} x .{ }^{\star} V(\mathbf{C})$ is the set of all internal sets. Let $A$ and $B$ be internal sets. Function $f: A \longrightarrow B$ is called internal if the graph of $f$ (i.e., $\{(x, f(x)) \mid x \in A\})$ is internal. $V(X)$ is called a countably saturated extension of $V(\mathbf{C})$ if it satisfies the following condition:

(Saturation Principle) If countable sequence of internal sets $A_{j} \in V(X) \backslash$ $X$ satisfies

$$
\bigcap_{j=1}^{k} A_{j} \neq \phi(k=1,2, \ldots)
$$

then

$$
\bigcap_{j=1}^{\infty} A_{j} \neq \phi .
$$

Let $A \in V(\mathbf{C}) \backslash \mathbf{C}$. From the Saturation Principle, we can show that if $A$ is an infinite set, ${ }^{s} A$ defined by

$$
{ }^{s} A=\left\{{ }^{\star} a \mid a \in A\right\}
$$


is a proper subset of ${ }^{\star} A$. Hence ${ }^{\star} A$ is an extended structure of $A$. ${ }^{\star} A$ is called the nonstandard extension of $\mathrm{A}$. By renaming the elements of $X$, we can assume without loss of generality that $\mathbf{C}$ is a subset of ${ }^{\star} \mathbf{C}$ and ${ }^{\star} x=x$ for each $x \in \mathbf{C}$.

Given any subset $U \subseteq V(\mathbf{C})$, define ${ }^{\star} U$ by

$$
{ }^{\star} U=\bigcup_{n \in \mathbf{N}}^{\star}\left(U \cap V_{n}(\mathbf{C})\right) .
$$

Let $F(\mathbf{C}) \subseteq V(\mathbf{C})$ be the set of finite sets, i.e.,

$$
F(\mathbf{C})=\{A \in V(\mathbf{C}) \backslash \mathbf{C} \mid A \text { is a finite set }\} .
$$

An element of ${ }^{\star} F(\mathbf{C})$ is called a hyperfinite set. It is shown that if $A$ is a hyperfinite set, then there is an initial segment $J=\left\{n \in{ }^{\star} \mathbf{N} \mid n \leq j\right\}$ for some $j \in{ }^{\star} \mathbf{N}$ and a one-to-one, onto internal mapping $f: J \longrightarrow A$. Thus, we will often write a hyperfinite set $A$ as $A=\left\{a_{1}, a_{2}, \ldots, a_{j}\right\}$, where $a_{k}=f(k), k \in J$.

Any element of ${ }^{\star} \mathbf{C}\left({ }^{\star} \mathbf{R}\right)$ is called a hypercomplex (hyperreal) number. We assume that ${ }^{\star} \mathbf{R} \subseteq{ }^{\star} \mathbf{C}$. ${ }^{\star} \mathbf{C}$ is a proper extension field of $\mathbf{C}$, and ${ }^{\star} \mathbf{R}$ is an ordered extension of $\mathbf{R}$. An element of ${ }^{\star} \mathbf{N}\left({ }^{\star} \mathbf{Z}\right)$ is called a hypernatural number (hyperinteger). A hypercomplex number $x$ is called infinite if $|x|>n$ for any $n \in \mathbf{N}$, finite, $|x|<\infty$, if there is some $n \in \mathbf{N}$ such that $|x|<n$, and infinitesimal if $|x|<\frac{1}{n}$ for any $n \in \mathbf{N}$.

For any $x, y \in{ }^{\star} \mathbf{C}$, we will write $x \approx y$ if $|x-y|$ is infinitesimal. For any finite hypercomplex number $x$, there is a unique complex number $z$ such that ${ }^{\star} z \approx x$; this $z$ is called the standard part of of $x$ and denoted by ${ }^{\circ} x$.

Let $\mathcal{A}$ be an internal normed linear space with norm $\|\cdot\|$. The principal galaxy $\operatorname{fin}(\mathcal{A})$ and the principal monad $\mu(0)$ are defined by

$$
\begin{gathered}
\operatorname{fin}(\mathcal{A})=\{x \in \mathcal{A} \mid\|x\|<\infty\} \\
\mu(0)=\{x \in \mathcal{A} \mid\|x\| \approx 0\}
\end{gathered}
$$

Both of them are linear spaces over $\mathrm{C}$. The nonstandard hull of $\mathcal{A}$ is the quotient linear space $\hat{\mathcal{A}}=\operatorname{fin}(\mathcal{A}) / \mu(0)$ equipped with the norm given by

$$
\left\|^{\circ} x\right\|={ }^{\circ}\|x\|
$$

for all $x \in \operatorname{fin}(\mathcal{A})$, where ${ }^{\circ} x=x+\mu(0)$. It is shown by the Saturation Principle that $\hat{\mathcal{A}}$ is a Banach space ${ }^{6}$.

Let $\nu$ be an infinite hypernatural number, i.e., $\nu \in{ }^{\star} \mathbf{N} \backslash \mathbf{N}$. Define ${ }^{\star} \mathbf{C}^{\nu}$ by

$$
{ }^{\star} \mathbf{C}^{\nu}=\left\{f \mid f:\{1, \ldots, \nu\} \rightarrow{ }^{\star} \mathbf{C} \text {; internal }\right\} .
$$


${ }^{\star} \mathbf{C}^{\nu}$ is a $\nu$-dimensional internal inner product space with the natural inner product and the internal norm $\|\cdot\|$ derived by the inner product. Define $\mathbf{M}=M\left({ }^{\star} \mathbf{C}, \nu\right)$, the set of $\nu \times \nu$ internal matrices over ${ }^{\star} \mathbf{C}$, by

$$
\mathbf{M}=\left\{f \mid f:\{1, \ldots, \nu\}^{2} \rightarrow{ }^{\star} \mathbf{C} \text {; internal }\right\} .
$$

Naturally $\mathbf{M}$ acts on ${ }^{\star} \mathbf{C}^{\nu}$ as the internal linear operators. Let $p_{\infty}$ be the operator norm on $\mathbf{M}$, i.e., $p_{\infty}(A)=\sup \left\{\|A \xi\| \mid\|\xi\| \leq 1, \xi \in{ }^{\star} \mathbf{C}^{\nu}\right\}$. Denote by $A^{*}$ the adjoint of $A \in \mathbf{M}$. Let $\tau$ be the internal normalized trace on $\mathbf{M}$, i.e.,

$$
\tau(A)=\frac{1}{\nu} \sum_{i=1}^{\nu} A_{i i}
$$

for $A=\left(A_{i j}\right) \in \mathbf{M}$. Then $\tau$ defines an internal inner product $(\cdot \mid \cdot)$ on $\mathbf{M}$ by $(A \mid B)=\tau\left(A^{*} B\right)$, for $A, B \in \mathbf{M}$. Its derived norm called the normalized Hilbert-Schmidt norm is denoted by $p_{2}$, i.e., $p_{2}(A)=\tau\left(A^{*} A\right)^{1 / 2}$ for $A \in \mathbf{M}$. Denote by $\left(\mathbf{M}, p_{\infty}\right)$ and $\left(\mathbf{M}, p_{2}\right)$ the normed linear spaces equipped with these respective norms. The principal galaxies $\operatorname{fin}_{\infty}(\mathbf{M})$ of $\left(\mathbf{M}, p_{\infty}\right)$ and $\operatorname{fin}_{2}(\mathbf{M})$ of $\left(\mathbf{M}, p_{2}\right)$ are defined as follows:

$$
\begin{aligned}
\operatorname{fin}_{\infty}(\mathbf{M}) & =\left\{A \in \mathbf{M} \mid p_{\infty}(A)<\infty\right\} \\
\operatorname{fin}_{2}(\mathbf{M}) & =\left\{A \in \mathbf{M} \mid p_{2}(A)<\infty\right\}
\end{aligned}
$$

The principal monads $\mu_{\infty}(0)$ of $\left(\mathbf{M}, p_{\infty}\right)$ and $\mu_{2}(0)$ of $\left(\mathbf{M}, p_{2}\right)$ are defined as follows:

$$
\begin{gathered}
\mu_{\infty}(0)=\left\{A \in \mathbf{M} \mid p_{\infty}(A) \approx 0\right\} \\
\mu_{2}(0)=\left\{A \in \mathbf{M} \mid p_{2}(A) \approx 0\right\} .
\end{gathered}
$$

The nonstandard hull $\hat{\mathbf{M}}_{2}=\mathrm{fin}_{2}(\mathbf{M}) / \mu_{2}(0)$ turns out to be a Hilbert space with inner product $\langle\cdot \mid \cdot\rangle$ and norm $\|\cdot\|_{2}$ defined by

$$
<A+\mu_{2}(0) \mid B+\mu_{2}(0)>={ }^{\circ}(A \mid B)
$$

and

$$
\left\|A+\mu_{2}(0)\right\|_{2}=p_{2}(A)
$$

for $A, B \in \operatorname{fin}_{2}(\mathbf{M})$.

The nonstandard hull $\hat{\mathbf{M}}_{\infty}=\operatorname{fin}_{\infty}(\mathbf{M}) / \mu_{\infty}(0)$ of $\left(\mathbf{M}, p_{\infty}\right)$ turns out to be a $C^{*}$-algebra equipped with norm $\hat{p}_{\infty}$ defined by

$$
\hat{p}_{\infty}\left(A+\mu_{\infty}(0)\right)={ }^{\circ} p_{\infty}(A)
$$

for $A \in \operatorname{fin}_{\infty}(\mathbf{M})$.

Hinokuma and Ozawa ${ }^{14}$ showed that another quotient space $\hat{\mathbf{M}}$ defined by

$$
\hat{\mathbf{M}}=\operatorname{fin}_{\infty}(\mathbf{M}) /\left(\mu_{2}(0) \cap \operatorname{fin}_{\infty}(\mathbf{M})\right) .
$$

is a von Neumann algebra of type $\mathrm{II}_{1}$ factor. 


\section{ACKNOWLEDGEMENTS}

The author expresses his appreciation to Professor M. Ozawa for his genaral supports.

${ }^{1}$ I.Ojima and M.Ozawa, "Unitary Representations of the Hyperfinite Heisenberg Group and the Logical Extension Methods in Physics", Open Systems and Information Dynamics. 2(1),107-128(1993).

${ }^{2}$ S.Gudder, "Toward a Rigorous Quantum Field Theory," Found.Phys. 24(9), 1205-1225(1994).

${ }^{3}$ M.Ozawa, "Phase operator problem and macroscopic extension of quantum mechanics," preprint (Nagoya University, 1992).

${ }^{4}$ T.Nakamura, "A Nonstandard Representation of Feynman's Path Integrals," J.Math.Phys. 32(2), 457-463(1991).

${ }^{5}$ A.Robinson, Non Standard Analysis(North-Holland, Amsterdam,1966).

${ }^{6}$ A.E.Hurd, and P.A.Loeb, An Introduction to Nonstandard Real Analysis(Academic Press,Orlando,1985).

${ }^{7}$ S.Gudder and V.Naroditsky, "Finite-Dimensional Quantum Mechanics," Int.J.Theor.Phys. 20(8),619-643(1981).

${ }^{8}$ A.O.Barut and A.J.Bracken, "Compact Quantum systems: Internal Geometry of Relativistic Systems," J.Math.Phys. 26(10),2515-2519(1985).

${ }^{9}$ R.J.B.Fawcett and A.J.Bracken, "Simple Orthogonal and Unitary Compact Quantum Systems and the Inönü-Wigner Contraction," J.Math. Phys.29(7),1521-1528(1988).

${ }^{10}$ D.T.Pegg and S.M.Barnett, "Phase properties of the quantized single-mode electromagnetic field," Phys.Rev.A 39(4),1665-1675(1989).

${ }^{11}$ F.T.Arecchi, et al., "Atomic Coherent States in Quantum Optics," Phys.Rev.A 6(6),2211-2237(1972).

${ }^{12}$ H.S.Green, "A generalized method of field quantization," Phys.Rev. 90(2),270(1953).

${ }^{13}$ Y.Ohnuki and S.Kamefuchi, Quantum Field Theory and Parastatistics (University of Tokyo Press,Tokyo,1982).

${ }^{14}$ T.Hinokuma and M.Ozawa, "Conversion from Nonstandard Matrix Algebras to Standard Factors of Type II 1 ," Illinois.J.Math. 37(1),1-13(1993).

${ }^{15}$ L.C.Moore,Jr., "Hyperfinite Extensions of Bounded Operators on a Separable Hilbert Space," Trans.Am.Math.Soc. 218,285-295(1976). 